\title{
A year in the life of German patients with COPD: the DACCORD observational study
}

\author{
This article was published in the following Dove Press journal: \\ International Journal of COPD \\ 20 July 2016 \\ Number of times this article has been viewed
}

Roland Buhl'
Carl-Peter Criée ${ }^{2}$
Peter Kardos ${ }^{3}$
Claus Vogelmeier ${ }^{4,5}$
Nadine Lossi ${ }^{6}$
Claudia Mailänder
Heinrich Worth
Hertent,
'Pulmonary Department, Mainz
University Hospital, Mainz,
${ }^{2}$ Department of Sleep and Respiratory
Medicine, Evangelical Hospital
Göttingen-Weende, Bovenden, ${ }^{3}$ Group
Practice and Centre for Allergy,
Respiratory and Sleep Medicine,
Red Cross Maingau Hospital,
Frankfurt am Main, ${ }^{4}$ Department of
Medicine, Pulmonary, and Critical
Care Medicine, University Medical
Center Giessen and Marburg,
Philipps-University Marburg, ${ }^{5}$ German
Center for Lung Research, Marburg,
${ }^{6}$ Clinical Research Primary Care,
Novartis Pharma GmbH, Nürnberg,
Facharztforum Fürth, Fürth, Germany

Correspondence: Roland Buhl

Pulmonary Department, Mainz University Hospital, Langenbeckstrasse I, 55 I3 I

Mainz, Germany

Tel +49 6I3I I7 7270

Email roland.buhl@unimedizin-mainz.de
Introduction: Randomized interventional trials generally recruit highly selected patients In contrast, long-term, noninterventional studies can reflect standard of care of real-life populations. DACCORD (Die ambulante Versorgung mit langwirksamen Bronchodilatatoren: COPDRegister in Deutschland [Outpatient Care With Long-Acting Bronchodilators: COPD Registry in Germany]) is an ongoing observational study, conducted in primary and secondary care in Germany, aiming to describe the impact of disease and treatments on real-life patients with chronic obstructive pulmonary disease (COPD).

Methods: Patients had a clinical and spirometry diagnosis of COPD, were aged $\geq 40$ years, and were initiating or changing COPD maintenance medication. The only exclusion criteria were asthma and participation in a randomized clinical trial. Exacerbation data were collected every 3 months. COPD medication, COPD Assessment Test, and forced expiratory volume in 1 second $\left(\mathrm{FEV}_{1}\right)$ were recorded at the end of the 1 year period.

Results: In the 6 months prior to baseline, $26.5 \%$ of the 3,974 patients experienced $\geq 1$ exacerbation, compared with $26.1 \%$ over the 1 -year follow-up (annualized rate 0.384 ). Importantly, only previous exacerbations and not poor lung function alone predicted an increased exacerbation risk. There was a general shift to lower disease severity from baseline to 1 year, predominantly as a consequence of a lower proportion of patients considered at high risk due to exacerbations. COPD Assessment Test mean change from baseline was -1.9 , with $48.9 \%$ of patients reporting a clinically relevant improvement. Overall persistence to medication was high, with $77.2 \%$ of patients still receiving the same class of medication at 1 year.

Conclusion: DACCORD suggests that in clinical practice, the large majority of COPD patients are symptomatic but seldom exacerbate and that widely used tools and treatment recommendations do not reflect this fully.

Keywords: COPD, epidemiology, COPD exacerbations

\section{Background}

Chronic obstructive pulmonary disease (COPD) is typically a progressive disease associated with substantial morbidity and mortality. ${ }^{1}$ According to the Global Initiative for Chronic Obstructive Lung Disease (GOLD), the two main goals for management of COPD are to reduce the current symptoms and to lower the risk of future exacerbations. ${ }^{1}$ The strongest evidence available of treatment effects on symptoms and exacerbation risk comes from randomized, interventional trials such as TORCH, UPLIFT, POET-COPD, and SUMMIT. ${ }^{2-5}$ Although these provide high-quality data, such trials generally recruit highly selected populations and are usually designed to demonstrate treatment efficacy on a specific disease characteristic. Data from their placebo arms are often described as representing "natural" disease progression, but they reflect neither a general COPD population nor the "real world" treatment of COPD, since patients are both highly selected and generally washed out of their maintenance medication on 
entry and are only permitted rescue medication or a limited selection of maintenance treatment. Furthermore, although the data from the active arms can be helpful in informing treatment decisions, they cannot always be generalized.

A second source of data is health care provider databases, often analyzed retrospectively. ${ }^{6-8}$ These can extract data from large cohorts over prolonged periods, with the results available relatively quickly. However, analyses depend on which information are captured in the databases. Researchers may therefore have to limit their studies to datasets that do not represent current standard of care (if a prolonged retrospective period is studied) or limit the population or end points, with the consequence that data may not be generalizable to broader populations. Data collected from prospective, longterm, noninterventional (observational) studies can be more representative of the current standard of care of real-life populations. Importantly, noninterventional studies permit the collection of standardized, high-quality data. DACCORD (Die ambulante Versorgung mit langwirksamen Bronchodilatatoren: COPD-Register in Deutschland [Outpatient Care With Long-Acting Bronchodilators: COPD Registry in Germany]) is an ongoing noninterventional study, and, to our knowledge, this is the largest such study on COPD to date. In this manuscript, we report the data of the patients who have completed the first year of the study period.

\section{Methods}

\section{Trial design}

DACCORD is an ongoing, longitudinal, prospective noninterventional study of 2-year duration, involving $\sim 6,000$ patients with COPD from 349 primary and secondary care practices distributed throughout Germany. Full details of the methods have been previously published. ${ }^{9}$

Specific visits are not mandated by the protocol, but, consistent with usual care in Germany, it was anticipated that data would be recorded approximately every 3 months. At the baseline visit, data collected from the Internet-based electronic case report forms included demographic and disease characteristics, prescribed COPD medication, COPD Assessment Test (CAT), exacerbations in the 6 months prior to entry (defined based on prescription of oral steroids and/or antibiotics or hospitalization), and forced expiratory volume in 1 second $\left(\mathrm{FEV}_{1}\right)$. At 3, 6 and 9 months, only exacerbation data were collected. At the 1-year visit, the collected data included prescribed COPD medication, CAT, exacerbations, and $\mathrm{FEV}_{1}$.

\section{Participants}

The main inclusion criteria were a diagnosis of COPD fulfilling the German COPD Disease Management Program (DMP) criteria (one of which is that COPD is confirmed by spirometry testing), age $\geq 40$ years, and initiating or changing COPD maintenance medication. Given the noninterventional nature of the study, the decision to initiate or change medication was made by the patients' physician prior to inclusion in DACCORD. In order to recruit as broad a population as possible, patients were excluded only if they were in the asthma DMP or if they were participating in a randomized clinical trial. The study is registered in the European Network of Centers for Pharmacoepidemiology and Pharmacovigilance (http://www.encepp.eu/encepp/ viewResource.htm?id=6316). The study was approved by the ethics committee of the University of Erlangen-Nuremberg. All patients provided written informed consent prior to inclusion.

\section{Objectives}

The main objective of the study is the documentation and description of the care of patients with COPD in Germany. For the 1-year analysis reported in this manuscript, specific objectives were:

1. To document exacerbations retrospectively (6 months preceding inclusion) and prospectively.

2. To measure patient-reported outcomes in terms of CAT.

3. To assess patient persistence to COPD maintenance treatment.

\section{Statistical methods}

The sample size - a total of 6,000 patients - was determined by a need to collect data that are representative of COPD management in Germany. There are $\sim 930$ respiratory specialists in Germany. We decided that representative coverage would be provided by inviting $\sim 300$ of this group to take part in the study (distributed throughout Germany), together with an additional 100 primary care physicians specializing in pulmonology. This would mean that each center would need to recruit an average of 15 patients.

Exacerbation rates were estimated using a negative binomial regression model with annualized numbers of exacerbation as dependent variable and no independent variable. For CAT total score, absolute changes from baseline are presented, together with the proportion of patients with clinically relevant (ie, $\geq 2$ unit) changes from baseline - either improvement or worsening. Medication persistence was analyzed at treatment class level, with patients considered "persistent" if they were receiving the same class of COPD maintenance treatment at the 1-year visit as at baseline; "switch" indicates that patients were receiving medication from a different class, whereas "add-on" is used for the patients who were receiving an additional class on top of the baseline class(es). The GOLD 2011 group was calculated on the basis of CAT total score. 
The main analyses were performed on the per-protocol population, which includes all patients in the recruited population who attended Visit 4 (the 1-year visit) and at least two of the three intermediate visits, and who had no relevant deviations from the observational plan. For reasons of data quality, the GOLD 2011 analyses exclude patients with a $>50 \%$ change in $\mathrm{FEV}_{1}$ between baseline and 1 year, as such changes were believed to be implausible.

\section{Results}

\section{Participants}

A total of 5,584 patients had valid baseline data and at least one item of postbaseline data, 4,259 of whom had attended the 12-month visit, with the per-protocol set including 3,974 patients (Figure 1). Of the 3,974 patients, 830 (20.9\%) newly initiated maintenance COPD medication on entry to DACCORD. The baseline demographics and disease characteristics are shown in Table 1, together with those of the 1,954 patients excluded from the analysis as they had no postbaseline data, did not complete the 1-year visit, or were excluded from the per-protocol population. The characteristics of both populations were consistent, with no clinically or statistically relevant differences.

\section{Outcomes}

\section{Exacerbations}

In the overall population, the annualized rate of exacerbations during the 1-year follow-up period was 0.384 (95\% confidence limit $0.356,0.413$ ). However, in patients with no exacerbation in the baseline period, the exacerbation rate was $0.259(0.236,0.284)$, whereas in patients with at least one baseline exacerbation, the rate during the follow-up period was $0.743(0.666,0.829)$. A total of 212 patients experienced two or more exacerbations during the 6-month baseline period; in these patients, the rate of exacerbations during the follow-up period was 1.230 (1.032, 1.467). Importantly, the annualized rate of exacerbations during the 1-year follow-up period was highest in GOLD D3 (ie, patients considered at high risk on the basis of exacerbations and lung function), while the rate in GOLD D1 (ie, high risk on the basis of lung function alone) was similar to the rate in GOLD A (Figure 2).

In the 6 months prior to study entry, 1,055 patients (26.5\%) experienced at least one exacerbation, whereas during the 1-year follow-up period, 1,036 patients (26.1\%) experienced at least one exacerbation (Figure 3 ). The majority of the patients with no exacerbations during the 6-month baseline period continued to experience no exacerbations over the 1-year follow-up period (Table 2). However, the majority of patients who exacerbated in the baseline period did not exacerbate during the 1-year follow-up. A total of $14.3 \%$ of patients either had missing exacerbations data at a visit or missed one visit during the 1-year period and had no exacerbations to report at the other visits. Of these patients, $77.4 \%$ did not exacerbate during the 6-month baseline period.

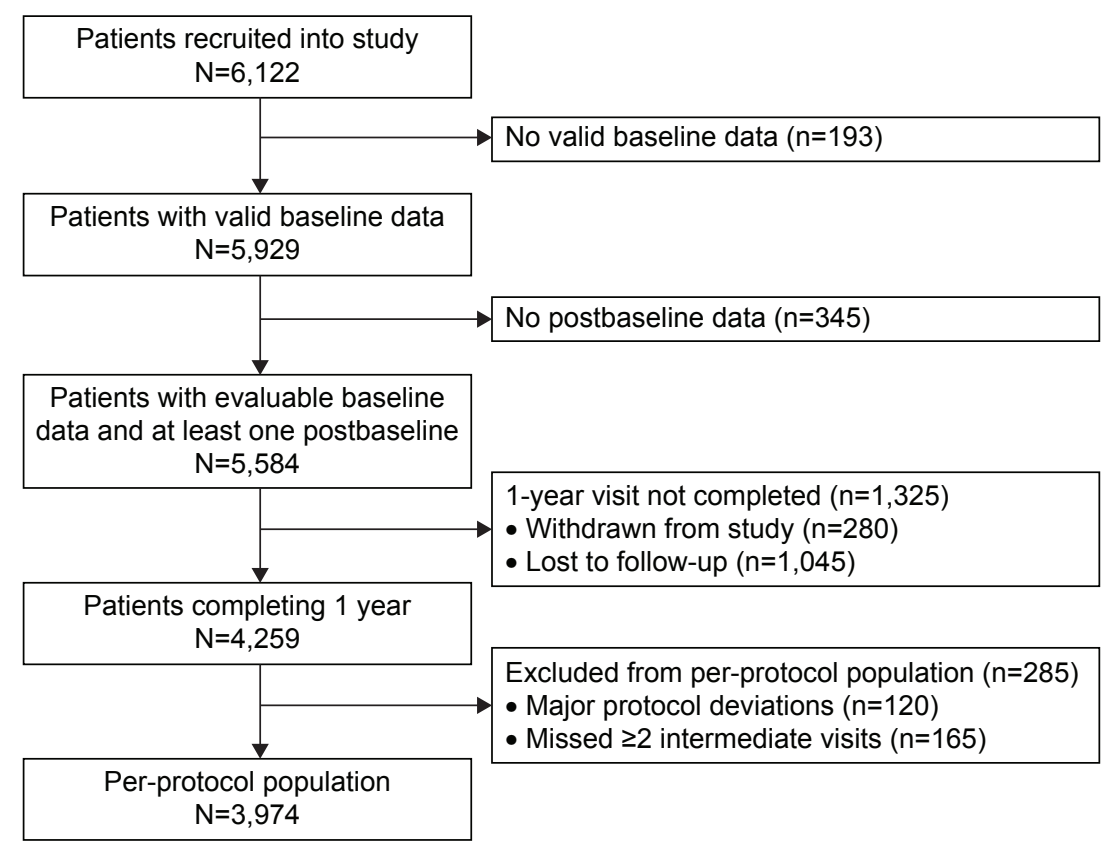

Figure I Cohort I patient flow through the first year of DACCORD.

Abbreviation: DACCORD, Die ambulante Versorgung mit langwirksamen Bronchodilatatoren: COPD-Register in Deutschland [Outpatient Care With Long-Acting Bronchodilators: COPD Registry in Germany]. 
Table I Baseline demographics and disease characteristics

\begin{tabular}{|c|c|c|}
\hline $\begin{array}{l}\text { Demographics and } \\
\text { characteristics }\end{array}$ & $\begin{array}{l}\begin{array}{l}\text { Overall } \\
\text { population } \\
(\mathrm{N}=3,974)\end{array} \\
\end{array}$ & $\begin{array}{l}\text { Excluded from per- } \\
\text { protocol population } \\
(\mathrm{N}=1,954)\end{array}$ \\
\hline \multicolumn{3}{|l|}{ Sex, n (\%) } \\
\hline Male & $2,362(59.4)$ & $\mathrm{I}, \mathrm{I} 76(60.2)$ \\
\hline Female & $1,612(40.6)$ & $774(39.8)$ \\
\hline Age (years), mean (SD) & $65.7(10.1)$ & $65.7(10.6)$ \\
\hline \multicolumn{3}{|l|}{ Age groups, $n(\%)$} \\
\hline$<65$ years & $\mathrm{I}, 809(45.5)$ & $903(46.2)$ \\
\hline $65-75$ years & $\mathrm{I}, 492(37.5)$ & $670(34.3)$ \\
\hline$>75$ years & $673(16.9)$ & $381(19.5)$ \\
\hline $\operatorname{BMI}\left(\mathrm{kg} / \mathrm{m}^{2}\right)$, mean $(\mathrm{SD})$ & $27.3(5.6)$ & $27.2(5.6)$ \\
\hline \multicolumn{3}{|c|}{ Duration since primary diagnosis, $\mathrm{n}(\%)$} \\
\hline$\leq$ I year & $963(24.2)$ & $619(31.7)$ \\
\hline$>$ I year & $3,011(75.8)$ & I,335 (68.3) \\
\hline \multicolumn{3}{|c|}{ FEV (percent predicted), ${ }^{a}$ n (\%) } \\
\hline$\geq 80$ & $733(18.4)$ & $355(18.2)$ \\
\hline 50 to $<80$ & $1,948(49.0)$ & $947(48.5)$ \\
\hline 30 to $<50$ & $1,066(26.8)$ & $544(27.8)$ \\
\hline$<30$ & $227(5.7)$ & $108(5.5)$ \\
\hline \multicolumn{3}{|c|}{ Smoking status at baseline, $\mathrm{n}(\%)$} \\
\hline Ex-smoker & $\mathrm{I}, 72 \mathrm{I}(43.3)$ & $690(35.3)$ \\
\hline Current smoker & I,479 (37.2) & $793(40.6)$ \\
\hline Never-smoker & $738(18.6)$ & $400(20.5)$ \\
\hline Missing data & $36(0.9)$ & $71(3.6)$ \\
\hline \multicolumn{3}{|c|}{ Symptoms at baseline, $n(\%)$} \\
\hline Yes & $3,860(97.1)$ & I,838 (94.I) \\
\hline No & III (2.8) & $91(4.7)$ \\
\hline Missing & $3(0.1)$ & $25(1.3)$ \\
\hline $\begin{array}{l}\text { mMRC dyspnea scale, } \\
\text { mean (SD) }\end{array}$ & $1.9(1.0)$ & $1.8(1.1)$ \\
\hline CAT score, mean (SD) & $20.2(7.6)$ & 19.5 (7.9) \\
\hline
\end{tabular}

Note: ${ }^{\text {R}}$ andom spirometry assessed without requirement for washout of COPD medication or additional inhalation of short-acting $\beta_{2}$-agonist.

Abbreviations: SD, standard deviation; BMI, body mass index; $\mathrm{FEV}_{1}$, forced expiratory volume in I second; mMRC, modified Medical Research Council; CAT, COPD Assessment Test.

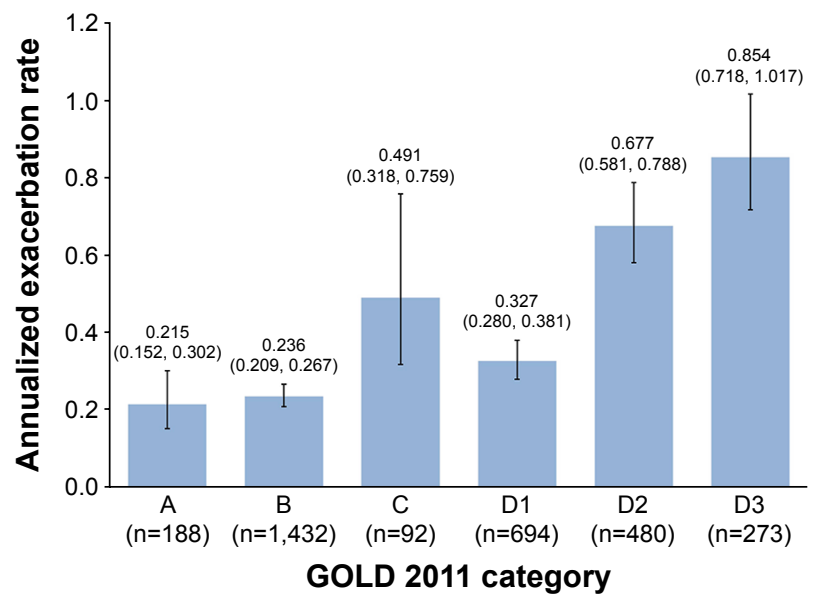

Figure 2 Annualized exacerbation rate (and 95\% Cl) during the I-year follow-up period, in patients grouped according to baseline GOLD 201 I category.

Notes: Due to low patient numbers, rates were not calculated for the GOLD C subgroups. GOLD A, low symptoms, low risk; GOLD B, high symptoms, low risk; GOLD C, low symptoms, high risk; GOLD D, high symptoms, high risk; GOLD DI, high risk on the basis of lung function alone; GOLD D2, high risk on the basis of exacerbations alone; GOLD D3, high risk on the basis of exacerbations and lung function. Abbreviation: GOLD, Global Initiative for Chronic Obstructive Lung Disease.

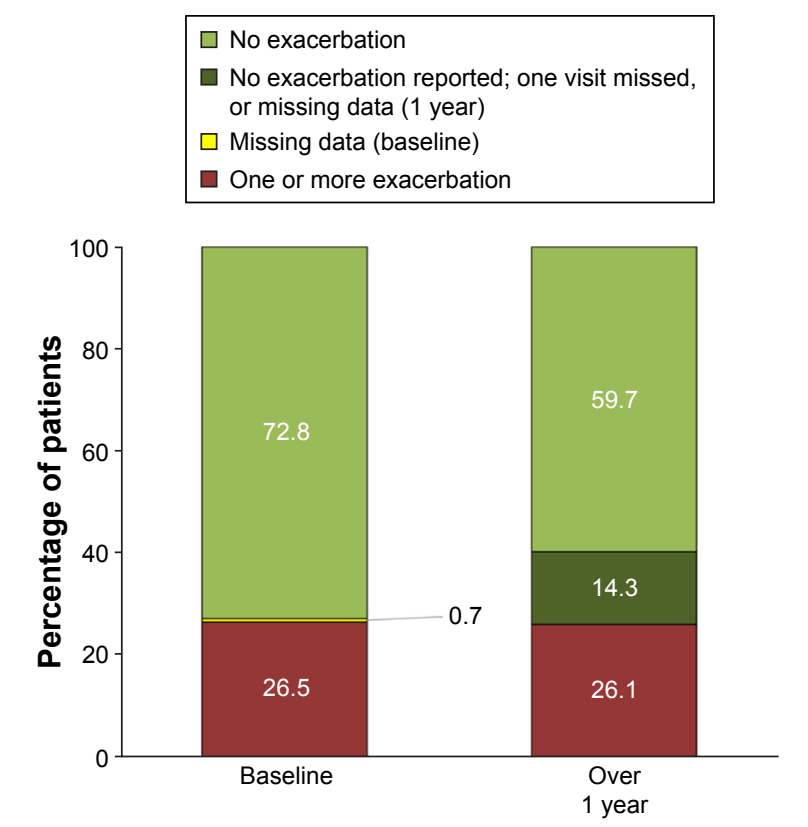

Figure 3 Percentage of patients reporting exacerbations at baseline and during the I year follow-up period (per-protocol population; $\mathrm{N}=3,974$ ).

\section{GOLD categorization}

In the GOLD 2011 categorization, there was a general shift from baseline to 1 year to lower grade, predominantly as a consequence of a lower proportion of patients considered at high risk due to exacerbations (with or without $\mathrm{FEV}_{1}$; Figure 4). However, the baseline GOLD 2011 categorization was based on the 6-month exacerbations data, extrapolated to 12 months.

\section{COPD Assessment Test}

The absolute change from baseline in mean CAT score is shown in Table 3. Overall, there was an improvement (ie, reduction) from baseline, which was just below the 2-unit level of clinical relevance. Nearly half (48.9\%) of the patients had a clinically relevant improvement from baseline in CAT total score at 1 year. The proportions of patients with no

Table 2 Number (\%) of patients reporting 0 , I, and $\geq 2$ exacerbations over I year ${ }^{\mathrm{a}}$ (per-protocol population; $\mathrm{N}=3,974$ )

\begin{tabular}{llll}
\hline Patients, $\mathbf{n}(\%)$ & \multicolumn{3}{l}{$\begin{array}{l}\text { Number of exacerbations during } \\
\text { the I-year follow-up }\end{array}$} \\
\cline { 2 - 4 } & $\mathbf{0}$ & $\mathbf{I}$ & $\geq \mathbf{2}$ \\
\hline $\begin{array}{l}\text { No exacerbations in the } \\
6 \text { months prior to baseline }\end{array}$ & $\mathrm{I}, 876(47.2)$ & $386(9.7)$ & $140(3.5)$ \\
$\begin{array}{l}\text { At least one exacerbation in } \\
\text { the } 6 \text { months prior to baseline }\end{array}$ & $460(11.6)$ & $219(5.5)$ & $176(4.4)$ \\
\hline
\end{tabular}

Note: ${ }^{a}$ The numbers reported in the table exclude patients with missing baseline data or with no exacerbation reported during the I-year follow-up, and patients with an exacerbation reported, but the number of exacerbations not reported. 


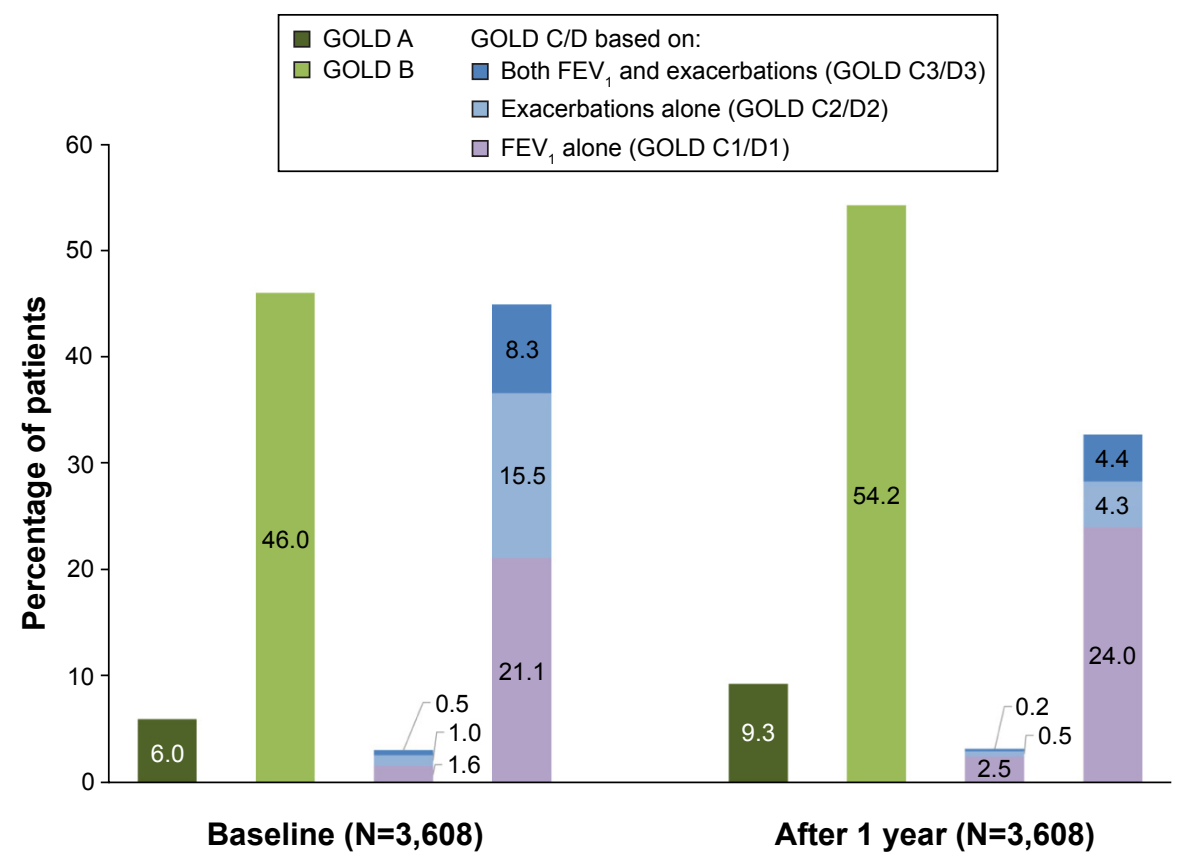

Figure 4 GOLD 20 I I categorization at baseline and after I year (per-protocol population).

Notes: Six patients in category $C$ and $2 \mathrm{I}$ in category $\mathrm{D}$ had insufficient data at the I-year visit to be assigned to a subcategory and so are not included. GOLD A, low symptoms, low risk; GOLD B, high symptoms, low risk; GOLD C, low symptoms, high risk; GOLD D, high symptoms, high risk; GOLD CI/DI, high risk on the basis of lung function alone; GOLD C2/D2, high risk on the basis of exacerbations alone; GOLD C3/D3, high risk on the basis of exacerbations and lung function.

Abbreviations: GOLD, Global Initiative for Chronic Obstructive Lung Disease; FEV , forced expiratory volume in I second.

change $(28.7 \%)$ and a clinically relevant worsening from baseline $(22.4 \%)$ were similar.

\section{COPD maintenance treatment persistence}

Overall persistence to treatment class was high, with 3,008 (77.2\%) of the 3,896 patients who had evaluable baseline medication data still receiving the same class of medication at the 1-year visit. In comparison, $11.9 \%$ of patients switched medication classes and $10.9 \%$ had added therapy from a different class at this visit. Figure 5 shows these data analyzed according to medication class at baseline. In the two most frequently prescribed groups, long-acting muscarinic antagonist (LAMA) monotherapy and long-acting $\beta_{2}$-agonist (LABA) plus LAMA plus inhaled corticosteroid (ICS), similar proportions of patients switched therapy. Of note, numerically fewer patients on LABA plus LAMA plus ICS

Table 3 CAT total score (per-protocol population; $\mathrm{N}=3,974$ )

\begin{tabular}{llll}
\hline & $\begin{array}{l}\text { Baseline CAT } \\
\text { total score }\end{array}$ & $\begin{array}{l}\text { CAT total score after } \\
\text { I year }\end{array}$ & \\
\cline { 2 - 4 } & & $\begin{array}{l}\text { Total } \\
\text { score }\end{array}$ & $\begin{array}{l}\text { Change from } \\
\text { baseline }\end{array}$ \\
\hline Mean (SD) & $20.2(7.6)$ & $18.3(7.8)$ & $-1.9(6.3)$ \\
Ist quartile, 3rd quartile & $15.0,26.0$ & $13.0,24.0$ & $-5.0,1.0$ \\
\hline
\end{tabular}

Abbreviations: CAT, COPD Assessment Test; SD, standard deviation. added on an additional medication class compared to those on LAMA monotherapy - as might be anticipated.

\section{Discussion}

DACCORD has recruited a large sample of COPD patients who required treatment intensification either as their first maintenance COPD treatment or as a change in their existing regimen (since only patients initiating or changing COPD maintenance medication were eligible). The majority had moderate airflow limitation at baseline, and almost all reported symptoms (as would be expected from the inclusion criteria). This is therefore a real-life population that is representative of patients with COPD who seek help in primary and secondary care.

Nearly $75 \%$ of patients in DACCORD did not exacerbate during the 1-year follow-up period, and the rate of exacerbations over this period was only 0.384 , suggesting that the majority of patients with COPD do not exacerbate, at least if treated with one (or more) long-acting bronchodilators. This is a group in which, unless their physician asks effective and appropriate questions, the symptom load and the true impact of COPD can be easily overlooked. The two key goals for the management of stable COPD are to reduce current symptoms and the risk of future exacerbations. ${ }^{1}$ The prevention of exacerbations has been considered the more important - which 


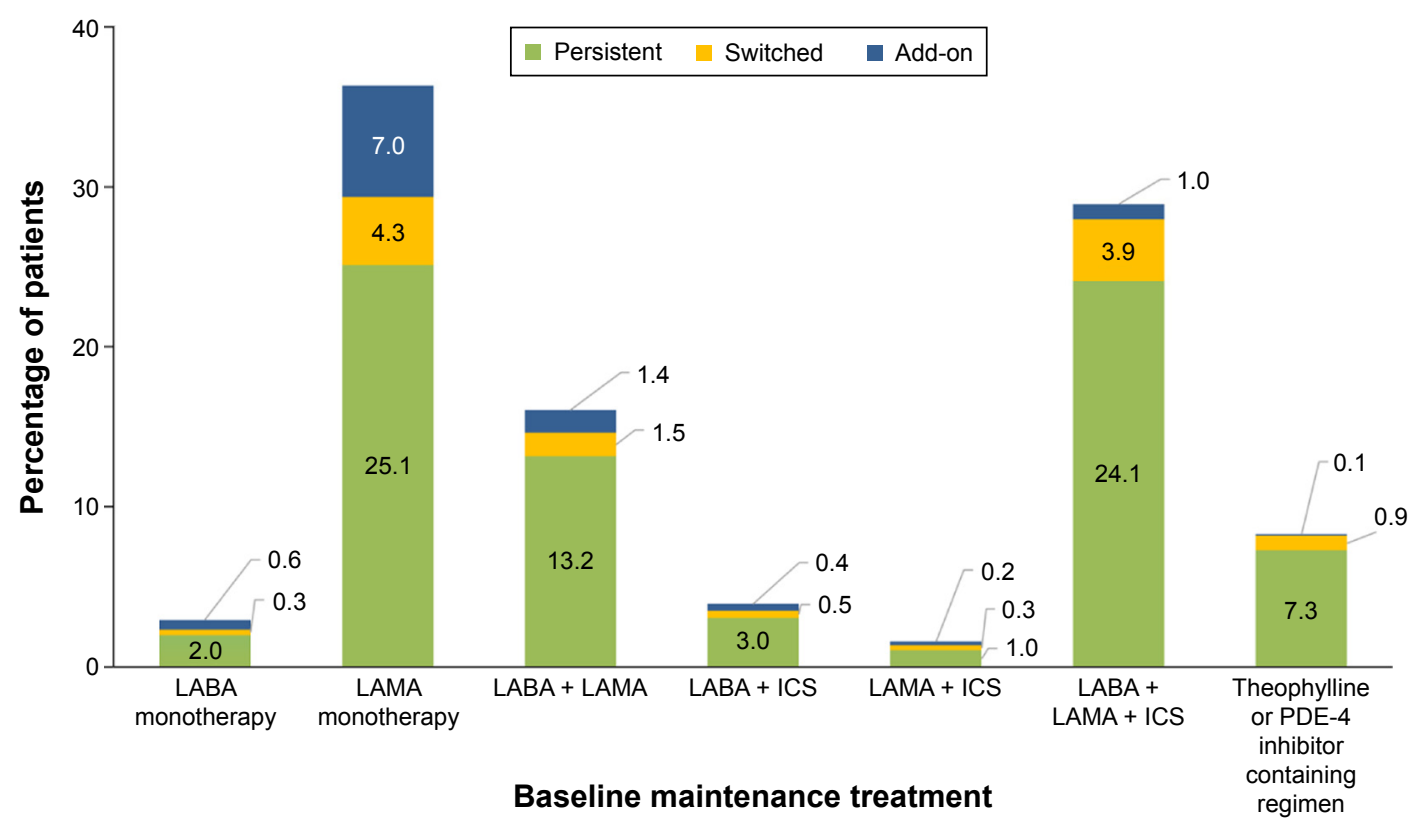

Figure 5 Percentages of patients persisting with, switching, or adding on medication classes at I year (per-protocol population). Note: $\mathrm{N}=3,974$ (data not evaluable for 78 patients).

Abbreviations: LABA, long-acting $\beta_{2}$-agonist; LAMA, long-acting muscarinic antagonist; ICS, inhaled corticosteroid; PDE-4, phosphodiesterase-4.

is understandable, given that exacerbations, especially those resulting in hospitalization, are responsible for much of the burden of COPD, ${ }^{10-15}$ and are associated with increased mortality. ${ }^{16}$ However, as shown in the PATHOS retrospective study, the annual rate of COPD exacerbations is decreasing - from 3.0 in 1999 to 1.3 in $2009 .{ }^{8}$ Other epidemiology studies have suggested that $>50 \%$ of the COPD population does not exacerbate; ${ }^{17-19}$ this is supported by the 3 -year ECLIPSE trial, in which on entry $53 \%$ of patients had not exacerbated in the previous year. ${ }^{20}$ Large randomized controlled trials also suggest a decrease over time in exacerbation rates: TRISTAN, published in 2003, reported on-treatment mean exacerbation rates of $0.97-1.05 ;{ }^{21} \mathrm{TORCH}$, published in 2007, had rates of $0.85-0.97 ;^{2}$ UPLIFT, published in 2008 , had rates of $0.73-0.85 ;^{3}$ and POET-COPD, published in 2011, had rates of $0.64-0.72 .{ }^{4}$ Even populations considered to be at increased risk of exacerbations do not necessarily exacerbate: the INVIGORATE study, conducted between 2009 and 2012, recruited patients with severe COPD and a history of at least one exacerbation in the previous year - so high risk according to GOLD 2011 (based on impaired lung function). ${ }^{22}$ During the 1-year study period, $62 \%$ of patients receiving either indacaterol $150 \mu \mathrm{g}$ or tiotropium $18 \mu \mathrm{g}$ once daily did not exacerbate. ${ }^{22}$ DACCORD, to our knowledge, is the first large, well-designed prospective noninterventional study to confirm these findings.

The consequence of guidelines emphasizing the importance of the prevention of exacerbations is that a large subset of patients are not optimally treated - for example, by the use of ICS to prevent exacerbations when they are not at increased risk of exacerbating, in turn exposing such patients to the recognized adverse effects of ICSs. ${ }^{23}$ This is especially true for patients in GOLD Group D, where the risk categorization has been shown to be predominantly driven by lung function..$^{24-27}$ This suggests that "lumping" the "poor lung function" Group D with the "exacerbation" Group D is misleading. Importantly, patients in DACCORD who were assigned to GOLD Group D on the basis of lung function alone (ie, Group D1) do not appear to be at increased risk of experiencing exacerbations over the 1-year follow-up. A logical conclusion is that, according to GOLD, ${ }^{1}$ reducing symptoms should be the main treatment goal for these patients.

A particular challenge with running a purely observational trial is that the only data captured are those generated from standard of care evaluations, with a limited ability to follow-up on any missing or substandard data. This means that there is a higher risk of missing data than from the more controlled environment of a randomized trial. These are limitations of DACCORD, and potential sources of bias, although typical issues with noninterventional, observational studies. In DACCORD, missing data had a particular impact on the exacerbations results, since in contrast to most of the data (which were captured only at the 12-month visit), exacerbations were analyzed across the full 1-year follow-up period. We hypothesize that if a patient experienced an exacerbation it would either trigger a visit to their physician 
(usually the investigator) and thus be documented or be recalled at the next visit. We suggest, therefore, that where a patient missed no more than one visit and reported "no" for exacerbations at the other visits, it is likely that they experienced no exacerbations for the duration of the study. This is supported by the observation that $77.4 \%$ of the patients with missing data had no exacerbation during the 6-month baseline period. If this is the case, then the "no exacerbation" phenotype remained stable for the duration of the study, with the majority of patients having not experienced an exacerbation in the 6 months prior to entering the study, and continuing to not experience an exacerbation during the first year of follow-up. Furthermore, although the overall rate of COPD exacerbations in the first year of DACCORD was low $(0.384)$, in the group of patients who did not exacerbate in the 6-month baseline period the rate of exacerbations during the follow-up period was even lower (0.259). This is consistent with an analysis of ECLIPSE, in which patients who did not exacerbate during the first year tended not to exacerbate in the subsequent 2 years. ${ }^{20}$ The selection of patients initiating or changing medication is another potential source of bias in this study. However, it is important to note that this was not a newly diagnosed population (only $25 \%$ of the population were diagnosed within the previous year), and only $20.9 \%$ newly initiated maintenance medication on entering the study.

CAT is a measure of health status that was developed for use in routine practice, partly to aid communication between physician and patient. ${ }^{28}$ It is relatively widely used in Germany, which is one of the reasons that it was selected for DACCORD, and is one of the symptom measures used for patient classification by GOLD. ${ }^{1}$ Although we recruited a population with a high symptom burden at baseline (97.1\% reporting at least one symptom on entry), this was not fully captured by CAT, with $\sim 10 \%$ of patients assigned to the low symptom GOLD Group A or C. The overall mean change from baseline in CAT total score was relatively small, just reaching clinical relevance. Nevertheless, approximately half of patients reported a clinically relevant improvement in health status (assessed by CAT), 1 year after a change in treatment. This emphasizes the importance of taking a more holistic approach to the care of patients with COPD, rather than just depending on a standardized (albeit useful) tool.

Medication persistence was analyzed at treatment class level, consistent with the approach of other similar studies. ${ }^{29,30}$ Compared with these previous studies, persistence was substantially higher in DACCORD (77.2\%). It is not clear why the persistence rate is so much higher in DACCORD. One possible reason is that these were two retrospective database studies, with persistence analyzed every 3 months, in which patients receiving medication on an intermittent basis would not necessarily be considered persistent. In contrast, by using only the 1-year data DACCORD may overestimate the degree of persistence. However, real-life studies generally provide more accurate persistence information than database studies. More relevant, perhaps, is that the population in DACCORD were not exacerbating, and (at a population level) their CAT score was improving, and so did not experience the major events that could trigger medication review (and intensification).

\section{Conclusion}

DACCORD suggests that in clinical practice, the large majority of COPD patients are symptomatic but seldom exacerbate and that widely used management and diagnostic tools, together with many treatment recommendations, do not necessarily reflect this fully.

\section{Acknowledgments}

The authors would like to thank the investigators and patients at the investigative sites for their support of this study. Writing support was provided by David Young of Young Medical Communications and Consulting Ltd. This support included the development of the first draft of the manuscript, under the guidance of the authors, and coordination of author comments and approval, and was funded by Novartis Pharma GmbH. This study was funded by Novartis Pharma GmbH.

\section{Disclosure}

Doctor R Buhl reports personal fees from AstraZeneca, Chiesi, GlaxoSmithKline, and Takeda and grants and personal fees from Boehringer Ingelheim, Novartis, and Roche. Doctor CP Criée reports personal fees from Boehringer Ingelheim, Chiesi, GSK, Novartis, Takeda, and Berlin-Chemie. Doctor P Kardos reports personal fees from Novartis, AstraZeneca, Boehringer Ingelheim, Chiesi, GSK, Menarini, and Takeda. Doctor C Vogelmeier reports personal fees from Almirall, AstraZeneca, Boehringer Ingelheim, Chiesi, GlaxoSmithKline, Janssen, Mundipharma, Novartis, and Takeda and grants and personal fees from Grifols. Doctor N Lossi is employed at Novartis Pharma $\mathrm{GmbH}$, Nürnberg, the sponsor of the study. Doctor C Mailänder is employed at Novartis Pharma GmbH, Nürnberg, the sponsor of the study. Doctor H Worth reports personal fees from AstraZeneca, Boehringer Ingelheim, Chiesi, GlaxoSmithKline, Klosterfrau, 
Menarini, Novartis, and Takeda. The authors report no other conflicts of interest in this work.

\section{References}

1. Global Initiative for Chronic Obstructive Lung Disease [homepage on the Internet]. Global strategy for the diagnosis, management, and prevention of chronic obstructive pulmonary disease; 2016. Available from: www.goldcopd.org. Accessed May 6, 2016.

2. Calverley PMA, Anderson JA, Celli B, et al. Salmeterol and fluticasone propionate and survival in chronic obstructive pulmonary disease. N Engl J Med. 2007;356(8):775-789.

3. Tashkin DP, Celli B, Senn S, et al. A 4-year trial of tiotropium in chronic obstructive pulmonary disease. $N$ Engl J Med. 2008;359(15): $1543-1554$.

4. Vogelmeier C, Hederer B, Glaab T, et al. Tiotropium versus salmeterol for the prevention of exacerbations of COPD. $N$ Engl J Med. 2011; 364(12):1093-1103.

5. Vestbo J, Anderson JA, Brook RD, et al. Fluticasone furoate and vilanterol and survival in chronic obstructive pulmonary disease with heightened cardiovascular risk (SUMMIT): a double-blind randomised controlled trial. Lancet. 2016;387(10030):1817-1826.

6. Punekar YS, Wurst K, Shukla A. Resource use and costs up to two years post diagnosis among newly diagnosed COPD patients in the UK primary care setting: a retrospective cohort study. COPD. 2015; 12(3):267-275.

7. James GD, Donaldson GC, Wedzicha JA, Nazareth I. Trends in management and outcomes of COPD patients in primary care, 2000-2009: a retrospective cohort study. NPJ Prim Care Respir Med. 2014;24:14015.

8. Ställberg B, Janson C, Johansson G, et al. Management, morbidity and mortality of COPD during an 11-year period: an observational retrospective epidemiological register study in Sweden (PATHOS). Prim Care Respir J. 2014;23(1):38-45.

9. Kardos P, Vogelmeier C, Buhl R, Criée C-P, Worth H. The prospective non-interventional DACCORD Study in the National COPD Registry in Germany: design and methods. BMC Pulm Med. 2015; 15(1):2.

10. Mackay AJ, Donaldson GC, Patel ARC, Jones PW, Hurst JR, Wedzicha JA. Usefulness of the chronic obstructive pulmonary disease assessment test to evaluate severity of COPD exacerbations. Am J Respir Crit Care Med. 2012;185(11):1218-1224.

11. Pitta F, Troosters T, Probst VS, Spruit MA, Decramer M, Gosselink R. Physical activity and hospitalization for exacerbation of COPD. Chest. 2006;129(3):536-544.

12. Solem CT, Sun SX, Sudharshan L, Macahilig C, Katyal M, Gao X. Exacerbation-related impairment of quality of life and work productivity in severe and very severe chronic obstructive pulmonary disease. Int J Chron Obstruct Pulmon Dis. 2013;8:641-652.

13. Blasi F, Cesana G, Conti S, et al. The clinical and economic impact of exacerbations of chronic obstructive pulmonary disease: a cohort of hospitalized patients. PLoS One. 2014;9(6):e101228.

14. Yu AP, Yang H, Wu EQ, Setyawan J, Mocarski M, Blum S. Incremental third-party costs associated with COPD exacerbations: a retrospective claims analysis. J Med Econ. 2011;14(3):315-323.
15. Punekar YS, Shukla A, Müllerova H. COPD management costs according to the frequency of COPD exacerbations in UK primary care. Int J Chron Obstruct Pulmon Dis. 2014;9:65-73.

16. Suissa S, Dell'Aniello S, Ernst P. Long-term natural history of chronic obstructive pulmonary disease: severe exacerbations and mortality. Thorax. 2012;67(11):957-963.

17. Uzaslan E, Mahboub B, Beji M, et al. The burden of chronic obstructive pulmonary disease in the Middle East and North Africa: results of the BREATHE study. Respir Med. 2012;106(suppl):S45-S59.

18. Andersson F, Borg S, Jansson SA, et al. The costs of exacerbations in chronic obstructive pulmonary disease (COPD). Respir Med. 2002;96(9): 700-708.

19. Soler-Cataluña JJ, Martínez-García MA, Román Sánchez P, Salcedo E, Navarro M, Ochando R. Severe acute exacerbations and mortality in patients with chronic obstructive pulmonary disease. Thorax. 2005;60(11):925-931.

20. Hurst JR, Vestbo J, Anzueto A, et al. Susceptibility to exacerbation in chronic obstructive pulmonary disease. N Engl J Med. 2010;363(12): $1128-1138$.

21. Calverley P, Pauwels R, Vestbo J, et al. Combined salmeterol and fluticasone in the treatment of chronic obstructive pulmonary disease: a randomised controlled trial. Lancet. 2003;361(9356):449-456.

22. Decramer ML, Chapman KR, Dahl R, et al. Once-daily indacaterol versus tiotropium for patients with severe chronic obstructive pulmonary disease (INVIGORATE): a randomised, blinded, parallel-group study. Lancet Respir Med. 2013;1(7):524-533.

23. Cates C. Inhaled corticosteroids in COPD: quantifying risks and benefits. Thorax. 2013;68(6):499-500.

24. Agusti A, Edwards LD, Celli B, et al. Characteristics, stability and outcomes of the 2011 GOLD COPD groups in the ECLIPSE cohort. Eur Respir J. 2013;42(3):636-646.

25. Han MK, Muellerova H, Curran-Everett D, et al. GOLD 2011 disease severity classification in COPDGene: a prospective cohort study. Lancet Respir Med. 2013;1(1):43-50.

26. Lange P, Marott JL, Vestbo J, et al. Prediction of the clinical course of chronic obstructive pulmonary disease, using the new GOLD classification: a study of the general population. Am J Respir Crit Care Med. 2012;186(10):975-981.

27. Haughney J, Gruffydd-Jones K, Roberts J, Lee AJ, Hardwell A, McGarvey L. The distribution of COPD in UK general practice using the new GOLD classification. Eur Respir J. 2014;43(4):993-1002.

28. Jones PW, Harding G, Berry P, Wiklund I, Chen W-H, Kline Leidy N. Development and first validation of the COPD assessment test. Eur Respir J. 2009;34(3):648-654.

29. Wurst KE, Punekar YS, Shukla A. Treatment evolution after COPD diagnosis in the UK primary care setting. PLoS One. 2014;9(9):e105296.

30. Penning-van BeestF, van Herk-Sukel M, Gale R, Lammers J-W, Herings R. Three-year dispensing patterns with long-acting inhaled drugs in COPD: a database analysis. Respir Med. 2011;105(2):259-265.
International Journal of COPD

\section{Publish your work in this journal}

The International Journal of COPD is an international, peer-reviewed journal of therapeutics and pharmacology focusing on concise rapid reporting of clinical studies and reviews in COPD. Special focus is given to the pathophysiological processes underlying the disease, intervention programs, patient focused education, and self management protocols.

\section{Dovepress}

This journal is indexed on PubMed Central, MedLine and CAS. The manuscript management system is completely online and includes a very quick and fair peer-review system, which is all easy to use. Visit $\mathrm{http}: / / \mathrm{www}$.dovepress.com/testimonials.php to read real quotes from published authors. 